(C) 2020 IEEE

Flexible Medium-Voltage Perturbation Injection Converter for AC and DC System Identification

M. Petkovic, S. Milovanovic, and D. Dujic

This material is posted here with permission of the IEEE. Such permission of the IEEE does not in any way imply IEEE endorsement of any of EPFL's products or services. Internal or personal use of this material is permitted. However, permission to reprint / republish this material for advertising or promotional purposes or for creating new collective works for resale or redistribution must be obtained from the IEEE by writing to pubs-permissions@ieee. org. By choosing to view this document, you agree to all provisions of the copyright laws protecting it. 


\title{
Flexible Medium-Voltage Perturbation Injection Converter for AC and DC System Identification
}

\author{
Marko Petković, Stefan Milovanović and Dražen Dujić
}

Power Electronics Laboratory, École Polytechnique Fédérale de Lausanne

marko.petkovic@epfl.ch; stefan.milovanovic@epfl.ch; drazen.dujic@epfl.ch

\begin{abstract}
Power systems have lately seen a tendency of increasing share of renewable energy sources and integration of power electronics equipment. The concept of distributed power generation introduces various stability-related challenges into the power system operation. Consequently, safe and reliable operation imposes the need to understand, describe and estimate the system stability through impedance-admittance measurements and identification. As in a present day network configuration the sources and loads can either be of ac or dc nature a flexible device capable of ac or dc measurements is required. The four-quadrant Cascaded H-Bridge topology features high output voltage resolution and high effective switching frequency which enables high-dynamic, high-fidelity voltage perturbation injection for medium voltage measurements. Moreover, through the hardware and control reconfiguration it is possible to address the needs of both ac and dc applications. This paper presents the flexible medium voltage Cascaded H-Bridge converter for impedance/admittance measurement of ac and dc systems. The effectiveness and flexibility of the topology is shown through a case study where the terminal characteristics of a Modular Multilevel Converter are measured.
\end{abstract}

Index Terms-dq-frame, Impedance Measurement, MediumVoltage, System Identification, Cascaded H-Bridge, MMC

\section{INTRODUCTION}

The increase in energy demand, combined with the advances in the field of power electronics has led to the deployment of renewable energy systems as well as broader installation of power conversion devices. Moreover, apart from the ac and high voltage $\mathrm{dc}(\mathrm{HVdc})$ used for energy distribution and transport, the potential of the medium voltage dc (MVdc) systems has been thoroughly analysed, however, there are no commercial solutions nor the standards yet available [1]-[5]. With the development of the MVdc systems comes the challenge of predicting their behaviour that is becoming increasingly complex. Due to the complexity of the installations, it becomes more challenging to model the system and predict possible instabilities that arise as a result of the interaction of different elements in the grid [6], [7]. Early identification and characterization of the present system and its future potential components would reduce the risk of encountering unpredictable behavior which would, in turn, provide a stable network with uninterrupted operation. This requires a mean to measure the impedance or admittance at different interfaces in the system, be it an ac or a dc interface.

However, the problem of high-power and medium voltage (MV) impedance/admittance measurement and system iden- tification had not yet been fully resolved and there is a lack of equipment capable of performing such measurements. The measurements in MV applications are not straightforward as there is a need for a device capable of operating at an MV level and injecting a perturbation into an MV system with a sufficient precision. At the same time having wide measurement bandwidth is sought after which is not easily achieved at MV level. The research performed in this field is scarce and the devices developed for that purpose are few. Medium voltage impedance/admittance estimators that exist today have been presented in [8]-[11]. These solutions have either limited bandwidth, up to $1 \mathrm{kHz}$ or comprise an output side transformer in order to step-up the voltage to the MV levels. This in turn also limits the output bandwidth. Moreover, all of these impedance estimators were reported using only an ac output. Still, the demand for such equipment is growing due to the need to support the development of recent MVdc and already established medium voltage ac (MVac) applications, grid integration of renewable energy sources and storage devices, energy transmission and distribution in the MV range and ensure safe integration with the existing apparatus.

One of the topologies capable of having sufficiently high voltage output and bandwidth is the Cascaded H-Bridge (CHB) topology with an active input element interfaced to a multi-winding transformer (MWT). This topology was already presented in [12], where the feasibility of using a multilevel topology for perturbation injection was studied. A high-frequency signal was superimposed to a fundamental component in open-loop and the admittance of a passive load was measured. The CHB converter is primarily intended to operate as an ac output converter. Nevertheless, it can be reconfigured without large effort to serve dc system purposes as well. Moreover, the CHB topology enables a bidirectional power flow, making it ideal for measurements of impedances or admittances of MVac or MVdc systems.

On the other side, to support the expansion of MVdc infrastructure, from the power electronics side, the modular multilevel converters (MMCs) have been used extensively [13] as they offer a high degree of flexibility in terms of power and voltage levels. However, from the equipment integration point of view there exists an issue when it comes to terminal characteristics, impedance and admittance, measurement of the MMCs [14]. Due to its flexibility, the CHB converter can be reconfigured and used to measure either the ac or the dc side 
characteristics of the MMC, which is taken as a case study here.

The rest of the paper is organized as follows. Section II presents the CHB converter and the reconfiguration possibilities, Section III presents the output voltage control, Section IV presents the measurement process and results using wideband signal perturbation injection while the Section $\mathrm{V}$ draws conclusions.

\section{CASCAded H-BRIdge IMPEDAnCE MEASUREMEnT UNIT}

The CHB converter, presented in Fig. 1, in the following analysis consists of a step-down MV-MWT with $15 \mathrm{sec}-$ ondary phase-shifted outputs permitting to arrange converter cells in an ac or a dc output structure through hardware reconfiguration. The MV-MWT in question has a primary side rated line voltage of $v_{1-1}^{p}=6 \mathrm{kV}$ and secondary side rated voltage of $v_{1-1}^{s}=710 \mathrm{~V}$. The MWT supplies threephase isolated voltages from its secondary, low-voltage, side to the cell, presented in Fig. 2. Isolated three-phase supply makes the cells and the dc-links independent from each other [15]. The input filter of the cell is the $L$-type filter with its parasitic resistance, which can be realized as a discrete inductor or through leakage inductance of the MWT [16]. The rectification of the input voltage is performed by the threephase Active Front End (AFE) switching at $f_{\mathrm{sw}}^{\mathrm{AFE}}=10 \mathrm{kHz}$. The supply voltage is rectified to $1.2 \mathrm{kV}$ at the dc-link. The dclink serves as an energy buffer and thus decouples the device under test (DUT) from the network the MWT is connected to. The AFE control system comprises a PLL that performs synchronisation to the primary side of the transformer, as it is essentially a grid-connected converter through the MWT [17]. The control is performed in a cascaded manner as the dc-link voltage is controlled through the input current control, which is implemented using the Synchronous Reference Frame (SRF) decoupled proportional integral (PI) control.

\section{A. Ac Output Configuration}

When the CHB converter is operated with an ac output, the phase-shifted transformer secondaries enable stacking up to 5 cells per phase and thus create a three-phase ac output with an increased output voltage levels and high effective switching frequency, provided a proper modulation scheme is used. This allows for higher output voltage bandwidth and thus higher frequency voltage perturbation injection and measurements. The switching frequency of an individual H-bridge (HB) equals $f_{\mathrm{sw}}^{\mathrm{HB}}=20 \mathrm{kHz}$. Using the bipolar phase-shifted PWM (PSPWM), by shifting the carriers modulating two neighboring cells, the equivalent switching frequency of the phase-leg can be increased to $N_{\text {cell }} \times f_{\mathrm{sw}}^{\mathrm{HB}}=f_{\mathrm{sw}}^{\mathrm{CHB}}=100 \mathrm{kHz}$. As a result, an outstanding performance and bandwidth can be ensured allowing for high-fidelity voltage perturbations to be injected into an unknown network or the DUT through an LC-type filter. The presence of the MWT on the input side is one of the advantages of this topology, at least from the output stage bandwidth point of view. Having the MWT at the input

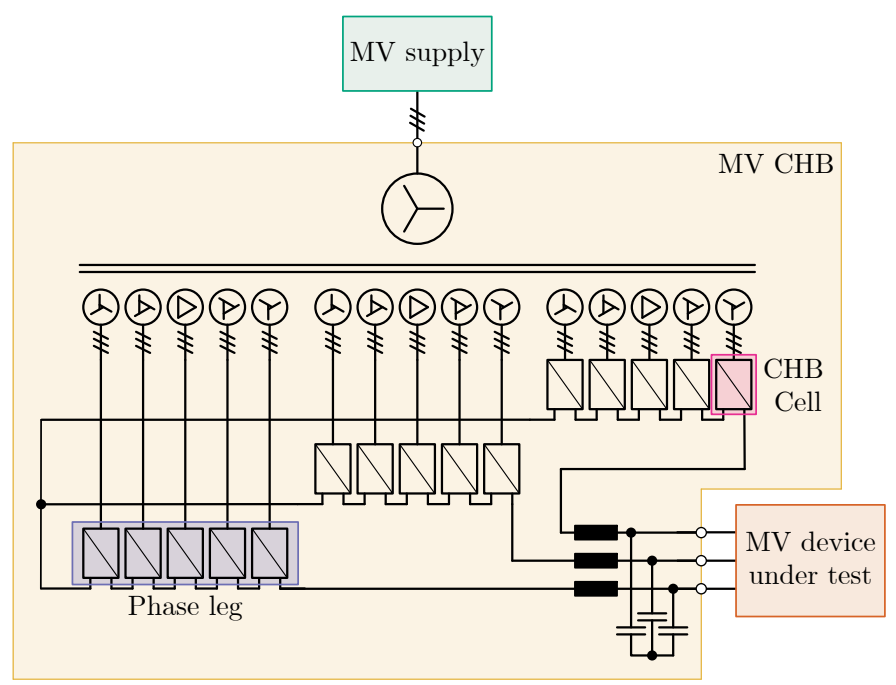

(a) $\mathrm{CHB}$ converter with an ac output configuration

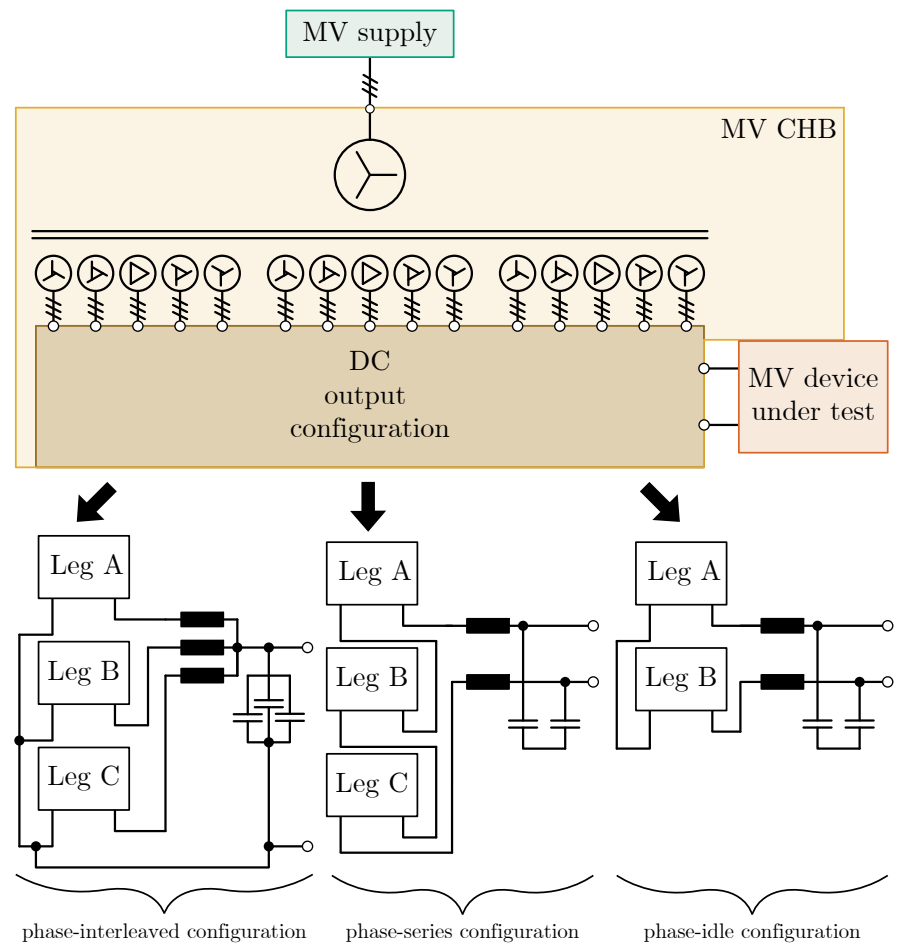

(b) CHB converter with a dc output configuration

Fig. 1. Cascaded H-Bridge topology for high dynamic medium-voltage perturbation injection and impedance measurement.

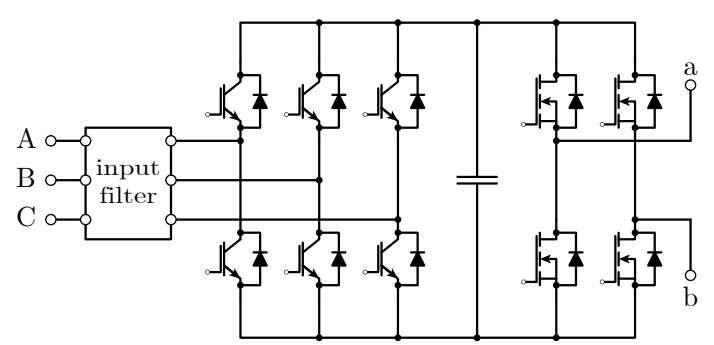

Fig. 2. Regenerative cell with the input filter and the AFE stage (left) and the inverter HB stage (right). 
means that there is no need to have a step-up transformer on the output side to elevate the voltage to MV level. As a result, the output stage high-frequency bandwidth is not limited by the leakage inductance of the output transformer. In an ac configuration the $\mathrm{CHB}$ cells outputs are interfaced to a threephase LC-type filter where the output voltage is controlled. This structure is presented in Fig. 1a. The converter is sized for a total dc-link voltage of $v_{\mathrm{dc}, \Sigma}=6 \mathrm{kV}$, per phase leg, and the power of $S_{\text {out }}^{\mathrm{CHB}}=1 \mathrm{MVA}$. Hence, the converter can be operated with an output voltage of anywhere between zero and $v_{\mathrm{out}}^{\mathrm{CHB}}=6 \mathrm{kV}$.

\section{B. Dc Output Configuration}

For the needs of measuring an impedance or admittance of a dc system, the $\mathrm{CHB}$ can be easily reconfigured to operate as a converter with a dc output voltage. When this mode of operation is sought after, there exist several possibilities of reconfiguration which can be presented with Fig. 1b. The modulation type is inherited from the ac output $\mathrm{CHB}$, i.e. the PS-PWM is used for the dc output as well.

A fairly simple and straightforward mean of achieving a dc operation with a $\mathrm{CHB}$ converter would be by interleaving individual phases into one single phase with a dc output. In this way the total dc-link voltage does not exceed the rated voltage and the output voltage is equal to $v_{\mathrm{out}}^{\mathrm{CHB}}=6 \mathrm{kV}$. Moreover, each phase shares $1 / 3$ of total current, thus preserving the original power rating. Furthermore, by interleaving the phase legs of the CHB the current ripple is additionally reduced. From the modulation point of view, the interleaving can be achieved by shifting the groups of carriers by $f_{\mathrm{sw}}^{\mathrm{CHB}} / 3$. This configuration is presented as phase-interleaved in Fig. $1 \mathrm{~b}$.

Another manner of obtaining a dc operation with the CHB converter is by connecting the phase legs, of a previously three-phase converter, in series and replacing the three-phase filter with a single-phase one. In this case the input voltage of the MV supply would need to be reduced in order to obtain a total dc-link voltage of $v_{\mathrm{dc}, \Sigma}=12 \mathrm{kV}$. This does not violate the voltage constraints of the converter as the MWT is designed to support voltage this high [17]. In turn, operating the converter with lower dc-link voltage leads to lower power processing capabilities as the rated power becomes reduced to $2 / 3$ of the initial one. Since the perturbation injection converters usually do not supply the main power of the system this does not arise as an issue. It would come as a problem only if the CHB would be used to supply the main power and the rated power of the DUT would surpass the $2 / 3$ of the CHB power. Such configuration is suitable for systems for which the output voltage of the phase-interleaved configuration is not sufficiently high to perform the perturbation injection. Eventually, the dc-link voltage could be elevated to $v_{\mathrm{dc}, \Sigma}=18 \mathrm{kV}$, provided that another transformer is used whose insulation is capable of withstanding this voltage. This proposition is presented as the phase-series configuration in Fig. 1b.

Finally, a third possibility is presented as phase-idle configuration in Fig. 1b. The advantage of the following proposition lies in the fact that it requires less hardware reconfiguration
TABLE I: Cell Parameters

\begin{tabular}{cc}
\hline Parameter & Value \\
\hline \hline Apparent Power Rating & $66.7 \mathrm{kVA}$ \\
AFE Nominal Current & $54 \mathrm{~A}$ \\
Inverter HB Nominal Current & $55.5 \mathrm{~A}$ \\
Filter Inductance & $150 \mu \mathrm{H}$ \\
Filter Capacitance & $3 \mu \mathrm{F}$ \\
Nominal dc-link voltage & $1200 \mathrm{~V}$ \\
Semiconductor blocking voltage & $1700 \mathrm{~V}$ \\
Inverter HB Modulation & PSC-PWM \\
\hline
\end{tabular}

TABLE II: Dc output configuration summary

\begin{tabular}{cccc}
\hline Phase configuration & $v_{\text {out }}^{\mathrm{CHB}}$ & $i_{\text {out }}^{\mathrm{CHB}}$ & $S_{\text {out }}^{\mathrm{CHB}}$ \\
\hline \hline interleaved & $6 \mathrm{kV}$ & $166 \mathrm{~A}$ & $1 \mathrm{MVA}$ \\
series & $12 \mathrm{kV}$ & $55.5 \mathrm{~A}$ & $667 \mathrm{kVA}$ \\
idle & $12 \mathrm{kV}$ & $55.5 \mathrm{~A}$ & $667 \mathrm{kVA}$ \\
\hline
\end{tabular}

and uses the original layout of the CHB topology. The output can be used in a way that only two out of three converter phases are utilized and the output is driven to be a dc output through the control. As in the previous case, in this case as well the output voltage is higher than in the first case. However, as only two out of three phases are used here the total power of the system is reduced to $2 / 3$ of the original power rating. An additional challenge is the operational state of the third phase leg. The simplest option stays to charge it through the AFE control and not operate the phase leg, i.e. not perform the modulation and switching. Different dc output configurations are summarized in Table II, while the typical CHB ac and dc output waveforms are presented in Fig. 3.

\section{Output Voltage Control}

The high equivalent switching frequency at the converter output is beneficial for the quality of the voltage output as well as the closed-loop bandwidth. In order to gain from this extended bandwidth single-loop voltage control is preferred over double-loop one. The reason is the fact doubleloop control limits the bandwidth as it is constrained by the bandwidth of the inner control loop and the output control loop bandwidth should be set up to ten times lower than the inner loop bandwidth. The bandwidth of the inner control loop, on the other hand, should be set below the $L C$ filter resonance frequency. In order to overcome this problem, the single-loop voltage control implemented is based on the method presented in [18] where the controller gains are obtained from system parameters and design specifications based on direct pole placement. The control method can be applied to the control of both ac and dc variables making it ideal for the needs of the CHB perturbation injection converter. Here, phase-interleaved configuration is taken as the dc output configuration. The control block diagram is presented in Fig. 4, while the gain values for ac and dc outputs are given in Table III. This 


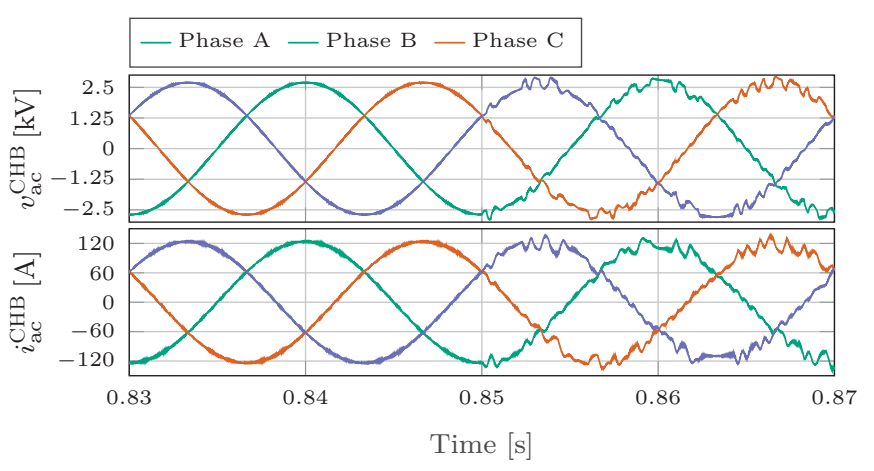

(a)

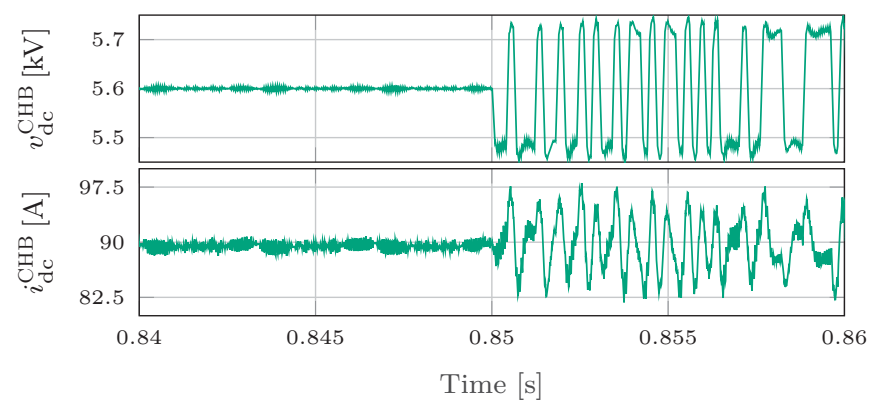

(b)

Fig. 3. Ac and dc voltage and current waveforms of the CHB before and after the start of the perturbation injection at $t_{s}=0.85 \mathrm{~s}$.

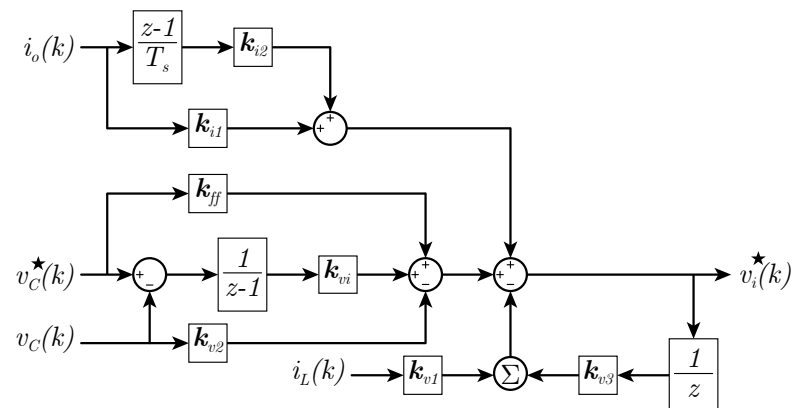

Fig. 4. Control block diagram of the output voltage controller.

TABLE III: State-space controller gains

\begin{tabular}{ccc}
\hline Gain & ac output & dc output \\
\hline \hline$k_{v 1}$ & $6.32-j 0.04$ & 9.1 \\
$k_{v 2}$ & $-0.1+j 0.0006$ & 0.3 \\
$k_{v 3}$ & $0.86-j 0.006$ & 0.98 \\
$k_{v i}$ & $0.0700+j 0.0034$ & 0.21 \\
$k_{f f}$ & $0.58+j 0.0156$ & 1.88 \\
$k_{i 1}$ & $15.17+j 0.035$ & 18.2 \\
$k_{i 2}$ & $(1.9754+j 0.005) \cdot 10^{-4}$ & $2 \cdot 10^{-4}$ \\
\hline
\end{tabular}

choice of gains results in a theoretical bandwidth of up to $f_{\mathrm{BW}}=7 \mathrm{kHz}$, following the developments provided in [18].

\section{MMC AC And DC Admittance Measurements}

In order to examine the effectiveness of the $\mathrm{CHB}$ as a perturbation injection converter, measurements of the input and output admittances of the MMC were simulated in PLECS simulation environment. The measurement setup can be illustrated with Fig. 5, while the operating parameters of the MMC are given in Table IV. It can be noticed that the measured input admittance is represented in the $d q$-frame. Even though the ac and $\mathrm{dc}$ rated voltages of the MMC are lower than the rated voltages of the $\mathrm{CHB}$, this does not pose an issue as the $\mathrm{CHB}$ converter can establish any voltage between zero and rated voltage at its terminals. The CHB converter is used to supply
TABLE IV: MMC Parameters

\begin{tabular}{cc}
\hline Parameter & Value \\
\hline \hline$S_{\text {nom }}$ & $500 \mathrm{kVA}$ \\
$\cos \phi$ & 1 \\
$v_{\mathrm{g}, 1-1}$ & $3.3 \mathrm{kV}$ \\
$i_{\mathrm{g}}$ & $87.5 \mathrm{~A}$ \\
$f_{g}$ & $50 \mathrm{~Hz}$ \\
$v_{\mathrm{dc}}$ & $5.6 \mathrm{kV}$ \\
$L_{\mathrm{br}}$ & $2.5 \mathrm{mH}$ \\
$C_{\mathrm{sm}}$ & $2.25 \mathrm{mF}$ \\
$f_{\mathrm{sw}, \mathrm{sm}}$ & $2 \mathrm{kHz}$
\end{tabular}

\# of submodules 8

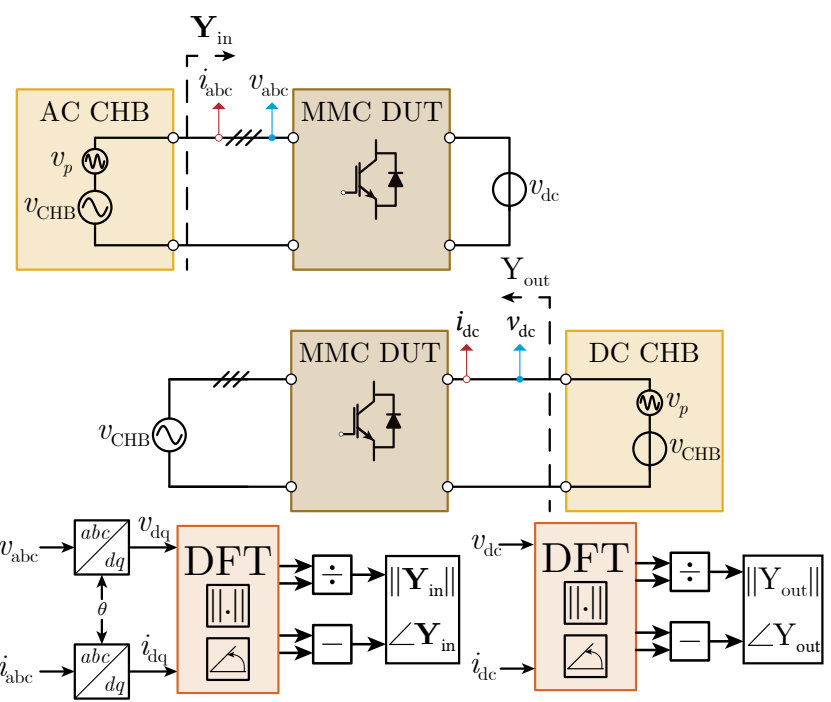

Fig. 5. Perturbation injection into an MV MMC and terminal characteristics measurement. 


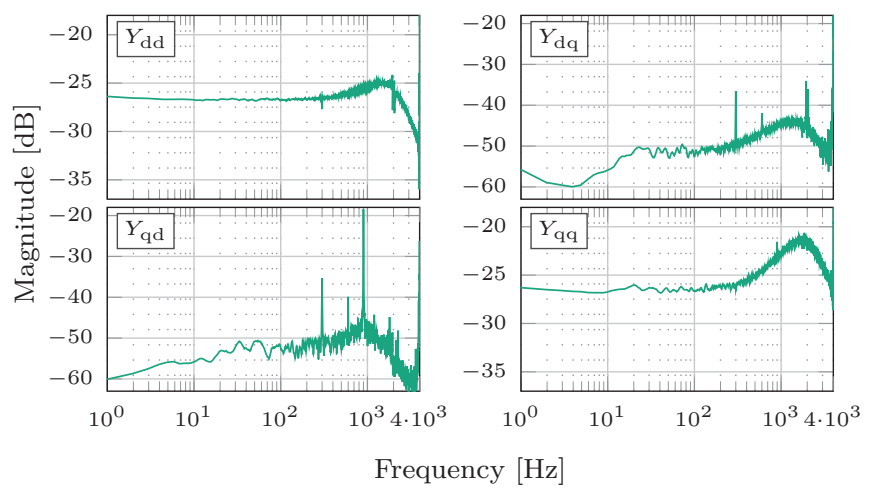

(a)

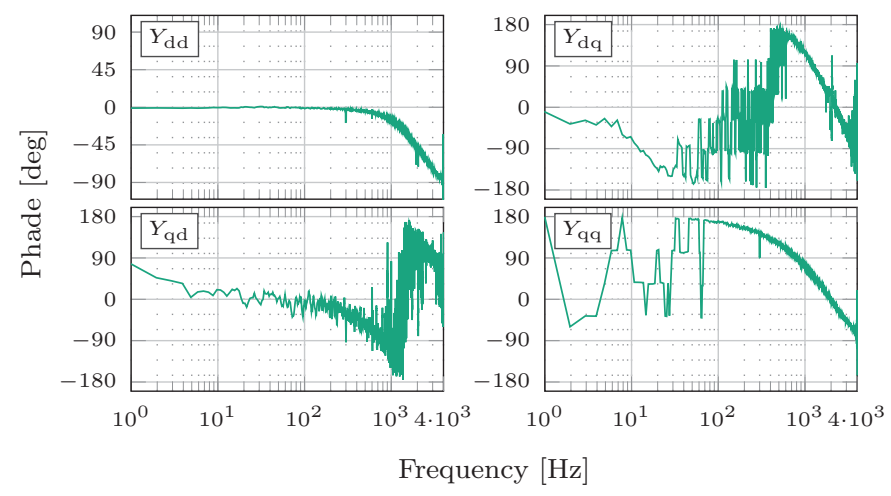

(b)

Fig. 6. a) magnitude and $\mathbf{b})$ phase of input impedance matrix $\mathbf{Y}_{\text {in }}$ of the MMC.

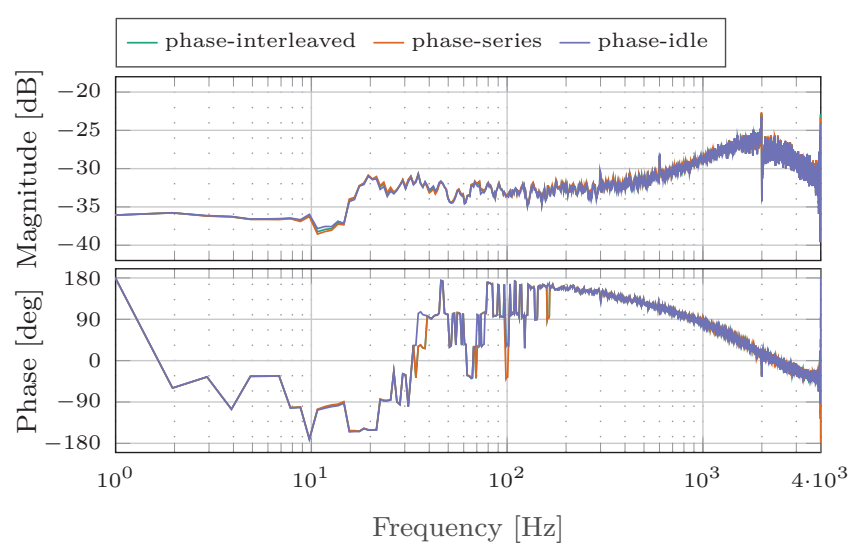

Fig. 7. Magnitude and phase of the output impedance $Y_{\text {out }}$ of the MMC.

the MMC either from the ac or from the dc side. Here, the $\mathrm{MMC}$ is operating as a grid-connected inverter and is driven either to supply the power to the CHB operating as an ac source or draw the power from the dc CHB. When supplying the $\mathrm{MMC}$ from the dc side the $\mathrm{CHB}$ can be reconfigured using one of the methods mentioned above. While interfaced to the MMC, from either the ac or the dc side, the CHB is at the same time driven to inject small-signal voltage perturbations into the MMC. The signal injected is a wideband pseudorandom binary sequence (PRBS) signal, namely the PRBS12 signal [19], with a generation frequency of $f_{\text {gen }}=4 \mathrm{kHz}$ which sets the frequency resolution of the measurement at about $f_{\text {res }}=1 \mathrm{~Hz}$. The use of such signals is beneficial due to the short time needed to perform the injection. This alleviates the problem of a DUT changing operational point during the perturbation which can especially happen in gridconnected applications. Following the perturbation injection, current and voltage sensors are used to measure the resulting current response, as well as the voltages at the ac and dc terminals of the MMC. Subsequently the measurements are collected and processed. A Discrete Fourier Transform (DFT) algorithm is performed to extract the frequency components from the current and voltage measurements. The admittances are then extracted as

$$
Y(s)=\frac{\|\tilde{i}(s)\|}{\|\tilde{v}(s)\|}[\angle \tilde{i}(s)-\angle \tilde{v}(s)]
$$

where $\tilde{v}(s)$ and $\tilde{i}(s)$ are the small-signal voltage perturbation and the resulting current response. This process is illustrated using Fig. 5.

The input and output admittances measured are presented in Figs. 6 and 7. From Fig. 6 it can be noticed that the diagonal terms of the input admittance matrix, $Y_{\text {in,dd }}$ and $Y_{\text {in,qq }}$, do not contain a large quantity of noise, whereas the off-diagonal elements are much less precise. This is due to the fact that the MMC was operated with power factor of $\cos \phi=1$ resulting in zero current in the $q$-axis, meaning it becomes difficult to discern the response to the perturbation from the simulation noise. The output admittance was measured using three dc output configurations presented in this work and it is seen that all three give notably similar results, which in the end should be the case as the reconfiguration mode should not affect the measurements.

\section{CONCLUSION}

This paper presents the flexible impedance/admittance measurement unit based on the CHB topology. Originally intended as an ac output converter, the $\mathrm{CHB}$ can be reconfigured to serve the purposes of measuring the characteristics of the dc systems as well. Depending on the converter voltage insulation constrains and power handling capabilities several reconfiguration methods can be applied, that have different levels of complexity. In terms of control methods, the ac output methods are effectively applied to the the control of dc variables, which simplifies the process of changing the mode of operation of the CHB converter. The use of efficient control methods permits the CHB unit to perform wideband signal injection and rapid measurements making it fitting for applications where the operating point of a DUT can change in a very short time. The MMC is taken as an example of a DUT that is supplied from both ac and dc sides by a CHB converter 
while simultaneously injecting small-signal perturbations into the MMC and measuring the ac and dc admittances. The solution put forward shows itself as a promising one for solving the issue of MV impedance/admittance measurements and an enabling technology for greater and faster integration of MVac and MVdc applications.

\section{REFERENCES}

[1] G. L. Kusic, G. F. Reed, J. Svensson, and Z. Wang, "A case for medium voltage dc for distribution circuit applications," in 2011 IEEE/PES Power Systems Conference and Exposition, IEEE, 2011, pp. 1-7.

[2] A. Hinz, M. Stieneker, and R. W. De Doncker, "Impact and opportunities of medium-voltage dc grids in urban railway systems," in 2016 18th European Conference on Power Electronics and Applications (EPE'16 ECCE Europe), IEEE, 2016, pp. 1-10.

[3] U. Javaid, F. D. Freijedo, W. van der Merwe, and D. Dujic, "Stability Analysis of Multi-Port MVDC Distribution Networks for All-Electric Ships," IEEE Journal of Emerging and Selected Topics in Power Electronics, pp. 1-1, 2019.

[4] M. Mogorovic and D. Dujic, "100 kW, 10 kHz MediumFrequency Transformer Design Optimization and Experimental Verification," IEEE Transactions on Power Electronics, vol. 34, no. 2, pp. 1696-1708, Feb. 2019.

[5] M. Utvic, S. Milovanovic, and D. Dujic, "Flexible Medium Voltage DC Source Utilizing Series Connected Modular Multilevel Converters," in 2019 21st European Conference on Power Electronics and Applications (EPE'19 ECCE Europe), 2019.

[6] X. Wang and F. Blaabjerg, "Harmonic Stability in Power Electronic Based Power Systems: Concept, Modeling, and Analysis," IEEE Transactions on Smart Grid, pp. 1-1, 2018.

[7] U. Javaid, F. D. Freijedo, D. Dujic, and W. Van Der Merwe, "Dynamic Assessment of Source-Load Interactions in Marine MVDC Distribution," IEEE Transactions on Industrial Electronics, vol. 64, no. 6, pp. 43724381, 2017.

[8] M. Jakšić, Z. Shen, I. Cvetković, D. Boroyevich, R. Burgos, C. DiMarino, and F. Chen, "Medium-Voltage Impedance Measurement Unit for Assessing the System Stability of Electric Ships," IEEE Transactions on Energy Conversion, vol. 32, no. 2, pp. 829-841, Jun. 2017.

[9] F. Hahn, S. Brüske, B. Benkendorff, G. Büticchi, F. W. Füchs, and M. Liserre, "Wide frequency range mediumvoltage grid impedance analysis by current injection of a multi-MW power converter," in 2016 18th Euro- pean Conference on Power Electronics and Applications (EPE'16 ECCE Europe), Sep. 2016, pp. 1-10.

[10] E. Ledezma, Kaiyu Wang, T. Keister, R. Edwards, R. Pipho, B. Palle, D. Kulkarni, T. E. Salem, J. C. Fox, and L. Parsa, "Development of a modular configurable multi-megawatt power amplifier," in IECON 2013 - 39th Annual Conference of the IEEE Industrial Electronics Society, Nov. 2013, pp. 631-636.

[11] F. Bogdan, J. Hauer, J. Langston, K. Schoder, M. Steurer, I. Cvetkovic, Z. Shen, M. Jaksic, C. DiMarino, F. Chen, D. Boroyevich, and R. Burgos, "Test environment for a novel medium voltage impedance measurement unit," in 2015 IEEE Electric Ship Technologies Symposium (ESTS), Jun. 2015, pp. 99-103.

[12] M. Petković, N. Hildebrandt, F. D. Freijedo, and D. Dujić, "Cascaded H-Bridge Multilevel Converter for a High-Power Medium-Voltage Impedance-Admittance Measurement Unit," in 2018 International Symposium on Industrial Electronics (INDEL), Nov. 2018, pp. 1-8.

[13] A. Lesnicar and R. Marquardt, "An innovative modular multilevel converter topology suitable for a wide power range," in 2003 IEEE Bologna Power Tech Conference Proceedings, IEEE, vol. 3, 2003, 6-pp.

[14] J. Lyu, X. Zhang, X. Cai, and M. Molinas, "Harmonic state-space based small-signal impedance modeling of a modular multilevel converter with consideration of internal harmonic dynamics," IEEE Transactions on Power Electronics, vol. 34, no. 3, pp. 2134-2148, 2018.

[15] Y. Li, Y. Wang, R. Wang, J. Wu, H. Zhang, Y. Feng, S. Li, W. Yao, and B. Q. Li, "Distributed Generation Grid-Connected Converter Testing Device Based on Cascaded H-Bridge Topology," IEEE Transactions on Industrial Electronics, vol. 63, no. 4, pp. 2143-2154, Apr. 2016.

[16] N. Hildebrandt and D. Dujic, "Supply Grid Synchronization and Operation of a Filterless Cascaded HBridge based Grid Emulator," in The 45th Annual Conference of the IEEE Industrial Electronics SocietyIECON 2019, 2019.

[17] N. Hildebrandt, M. Luo, and D. Dujic, "Robust and cost effective synchronization scheme for a multicell grid emulator," IEEE Transactions on Industrial Electronics, pp. 1-1, 2020.

[18] H.-S. Kim, H.-S. Jung, and S.-K. Sul, "Discrete-time voltage controller for voltage source converters with lc filter based on state-space models," IEEE Transactions on Industry Applications, vol. 55, no. 1, pp. 529-540, 2018.

[19] K. Godfrey, Perturbation signals for system identification. Prentice Hall International (UK) Ltd., 1993. 